\title{
Wild monodromy and automorphisms of curves *
}

\author{
Claus Lehr and Michel Matignon
}

July 25, 2018

\begin{abstract}
Let $R$ be a complete discrete valuation ring of mixed characteristic $(0, p)$ with field of fractions $K$ containing the $p$-th roots of unity. This paper is concerned with semi-stable models of $p$-cyclic covers of the projective line $C \longrightarrow \mathbb{P}_{K}^{1}$. We start by providing a new construction of a semi-stable model of $C$ in the case of an equidistant branch locus. If the cover is given by the Kummer equation $Z^{p}=f\left(X_{0}\right)$ we define what we called the monodromy polynomial $\mathcal{L}(Y)$ of $f\left(X_{0}\right)$; a polynomial with coefficients in $K$. Its zeros are key to obtaining a semi-stable model of $C$. As a corollary we obtain an upper bound for the minimal extension $K^{\prime} / K$ over which a stable model of the curve $C$ exists. Consider the polynomial $\mathcal{L}(Y) \prod\left(Y^{p}-f\left(y_{i}\right)\right)$ where the $y_{i}$ range over the zeros of $\mathcal{L}(Y)$. We show that the splitting field of this polynomial always contains $K^{\prime}$, and that in some instances the two fields are equal.
\end{abstract}

\section{Introduction}

Let $R$ be a complete discrete valuation ring of mixed characteristic $(0, p)$ with field of fractions $K$ containing the $p$-th roots of unity. This work is about semi-stable models of $p$-cyclic covers of the projective line $\mathbb{P}_{K}^{1}$. It is a continuation of a series of papers on this topic that began with work of Coleman and McCallum who used rigid geometry in $\mathrm{Co}$ and $\mathrm{Co}-\mathrm{Mc}$. Our approach is closer to Raynaud's ideas introduced in Ra1. Both [Le] and [Ma] dealt with computational aspects of the semi-stable models of such a cover $C \longrightarrow \mathbb{P}_{K}^{1}$ in the case that the branch locus $B$ consists of $K$-points and has equidistant geometry. The latter means that $\mathbb{P}_{K}^{1}$ has a smooth $R$-model with the property that the points of $B$ have pairwise distinct specializations on the special fiber. In previous work little attention has been given to the determination of the finite monodromy, i.e. the minimal extension $K^{\prime} / K$ such that $C_{K^{\prime}}$ has a semi-stable model. Let $l \geq 3$ be a prime different from $p$. It is known (cf. Des Thm.5.15) that the extension $K^{\prime} / K$ is a certain subextension of the minimal extension over which the $l$-torsion points of the jacobian $J(C)$ are rational. The tame part of $K^{\prime} / K$ has been described in a more general setting by Liu and Lorenzini in [Li-Lo. In [Si-Za1] and Si-Za2 Silverberg and Zarhin study the finite monodromy groups which can occur for abelian varieties over a local field of residue characteristic $p \geq 0$. In the case of abelian surfaces they are able to list these groups in the equal characteristic $p>0$ case.

*Work supported in part by the European Community's Sixth Framework Programme under Contract MRTN-CT2003-504917 
So far, in mixed characteristic, there are few techniques available to study the wild finite monodromy, i.e. the extension corresponding to the Sylow $p$-subgroup of $\operatorname{Gal}\left(K^{\prime} / K\right)$. The literature contains mostly cases where $\operatorname{deg}\left(\left[K^{\prime}: K\right]\right)$ is strictly divisible by $p$. An exception to this is the work of Krir concerning modular curves (cf. $[\mathrm{Kr}] \mathrm{Thm} .2$ ). This paper is concerned with the wild finite monodromy for $p$-cyclic covers in mixed characteristic $(0, p)$.

In section 2, we review the definitions of the semi-stable models we shall be concerned with. Associated with $C / K$ are the finite monodromy and related Galois groups which will be discussed briefly.

Section 3 is the technical heart of the paper. Here, we introduce the monodromy polynomial $\mathcal{L}(Y)$. If the cover is given birationally by the Kummer equation $Z^{p}=$ $f\left(X_{0}\right)$ then $\mathcal{L}(Y)$ is related to the logarithmic derivative $f^{\prime}(Y) / f(Y)$. The definition of $\mathcal{L}(Y)$ is key to the construction of the stable model as given in Theorem [5.1. The novelty with respect to the previous work of the authors is that the construction of the semi-stable model we give here is very well adapted to study the finite monodromy. In [Ma a polynomial with similar properties as $\mathcal{L}(Y)$ was defined. The zeros of either polynomial are centers for the blowups yielding a stable model of $C$. Yet the polynomial given in Ma has very high degree which allows it to be used for monodromy purposes only in cases of small genus.

Next, in section 4, we provide the needed results on the degeneration of $\mu_{p}$-torsors. The way they are stated here is with a view towards the later use in conjunction with the monodromy polynomial and equidistant geometry of the branch locus. Remark 4.3 explains this connection in some detail and we will often refer to it later.

Section 5 contains the main result, Theorem 5.1. It is a new characterization of semi-stable models of $p$-cyclic covers of the affine line using the monodromy polynomial Corollary 5.2 is an important step towards understanding the finite monodromy extension, i.e. the minimal extension over which $C$ has a stable model.

The Galois group of the finite monodromy acts on the special fiber $C_{R^{\prime}} \otimes_{R^{\prime}} k$ of the stable model:

$$
\operatorname{Gal}\left(K^{\prime} / K\right) \hookrightarrow \operatorname{Aut}\left(C_{R^{\prime}} \otimes_{R^{\prime}} k\right)
$$

The bounds derived from [Le-Ma1] Theorem 1.1 for the right hand side also limit the possible size of $\operatorname{Gal}\left(K^{\prime} / K\right)$. Hence one can ask if $v_{p}\left(\left|\operatorname{Gal}\left(K^{\prime} / K\right)\right|\right)$ can be maximal? (Here $v_{p}$ is the $p$-adic valuation with $v_{p}(p)=1$ ). In section ?? we give some examples concerning this question for covers that are defined over $\mathbb{Q}_{p}^{\text {tame }}$. The reason for working over this ground field is that we are mostly interested in the wild part of the monodromy. We describe various situations where the above can be affirmatively answered. (cf. Examples [5.5 and [5.6). The proofs for this section and more on maximal monodromy will appear in Le-Ma2.

\section{Stable models and finite monodromy}

Let $R$ be a complete mixed characteristic $(0, p)$ discrete valuation ring with field of fractions $K$ and algebraically closed residue field $k$. Denote by $v$ the valuation defined by $R$ on $K$ and by $\pi$ a uniformizer. We will always assume that $R$ contains a primitive $p$-th root of unity $\zeta$ and define $\lambda=\zeta-1$. Then $v\left(\lambda^{p-1}\right)=v(p)$. Let $C \longrightarrow \mathbb{P}_{K}^{1}$ be a $p$-cyclic cover of smooth projective geometrically irreducible $K$-curves with genus $(C) \geq 2$. We assume that the branch locus $B$ of the cover consists of 
$K$-points, write $m=|B|-1$, and assume that $B$ has equidistant geometry in the sense of the following definition.

Definition 2.1. We say that $B$ has equidistant geometry if there exists a smooth $R$-model $\operatorname{Proj}(R[u, w])$ for $\mathbb{P}_{K}^{1}$ such that the points of $B$ have distinct specializations on the special fiber of the model.

We can assume that, with respect to the coordinate $X_{0}:=u / w$, the cover is given birationally by the equation $Z_{0}^{p}=f\left(X_{0}\right)$ with $f\left(X_{0}\right) \in R\left[X_{0}\right]$ monic, $n=\operatorname{deg}\left(f\left(X_{0}\right)\right)$, $(n, p)=1$ and $n \leq m(p-1)$. Then any two distinct zeros of $f\left(X_{0}\right)$ specialize to distinct elements in $k$. We denote by $K(C) / K\left(X_{0}\right)$ the function field extension corresponding to the cover $C \longrightarrow \mathbb{P}_{K}^{1}$. The notion of an equidistant branch locus already appears in the literature. We refer to [Ra2, Gr-Ma and $\mathrm{Ma}$ for more on this.

\subsection{Stably marked models and finite monodromy}

For the basic definitions and generalities concerning semi-stable models, we refer to the article of Abbes $\mathrm{Ab}$.

The following result is due to Deligne and Mumford (cf. De-Mu Cor.2.7 and Des Prop.5.7, Lemme 5.16) and is true also in a more general setting.

Theorem 2.2 (Deligne-Mumford). With the above notation there exists a minimal extension $K^{\prime} / K$ such that $C_{K^{\prime}}$ has a stable model $C_{R^{\prime}}^{\circ}$ over the integral closure $R^{\prime}$ of $R$ in $K^{\prime}$. Further $K^{\prime} / K$ is Galois and acts faithfully on the special fiber:

$$
\operatorname{Gal}\left(K^{\prime} / K\right) \hookrightarrow \operatorname{Aut}_{k}\left(C_{R^{\prime}}^{\circ} \otimes_{R^{\prime}} k\right)
$$

In accordance with Raynaud (cf. Ra2 $\S 4$ ) we make the following definition.

Definition 2.3. Let $K^{\prime} / K$ be the minimal extension over which $C$ has a stable model. We call $\operatorname{Gal}\left(K^{\prime} / K\right)$ the finite monodromy group associated to $C$. The extension $K^{\prime} / K$ is called the finite monodromy extension. $\operatorname{Gal}\left(K^{\prime} / K\right)$ has a unique $p$-Sylow subgroup which we call the wild monodromy group $\operatorname{Gal}\left(K^{\prime} / K\right)_{w}$. The field extension $K^{\prime} / K^{\prime \operatorname{Gal}\left(K^{\prime} / K\right)_{w}}$ is called the wild monodromy extension.

Keeping the above notation, the following is a consequence of [Liu1] Cor.2.20.

Proposition 2.4. Let $K^{\prime} / K$ be the finite monodromy extension as in Definition 2.3. Then there exists a semi-stable model $C_{R^{\prime}}$, defined over the integral closure $R^{\prime}$ of $R$ in $K^{\prime}$ minimal with the property that the points in the ramification locus of $C_{K^{\prime}} \longrightarrow \mathbb{P}_{K^{\prime}}^{1}$ specialize to distinct smooth points on the special fiber. Further the finite monodromy group operates faithfully on its special fiber:

$$
\operatorname{Gal}\left(K^{\prime} / K\right) \hookrightarrow \operatorname{Aut}_{k}\left(C_{R^{\prime}} \otimes_{R^{\prime}} k\right)
$$

Definition 2.5. The semi-stable model $C_{R^{\prime}}$, given by Proposition 2.4 is called the stably marked model. We refer to the component to which the ramification points specialize as original component. Any model obtained from the stably marked model by a base change to a ring $R^{\prime \prime} \supset R^{\prime}$ we refer to as a stably marked model.

The next result is due to Raynaud (cf. Ra1 Thm.2). For an effective proof we refer to [Ma], Thm.3.2.2. 
Proposition 2.6. Let $C \longrightarrow \mathbb{P}_{K}^{1}$ be as above. Then the dual graph of the special fiber of a stably marked model of $C$ is an oriented tree whose origin corresponds to the original component (cf. Def[2.5). (For short we say that the special fiber is tree-like).

Proposition 2.7. Let $C \longrightarrow \mathbb{P}_{K}^{1}$ be as above. Assume that for each irreducible component $E$ of genus $>0$ in the stable reduction there exists a flat quasi-projective $R$-model $\mathcal{C}$ for $C$, such that $\mathcal{C} \otimes_{R} k$ is birational to $E$. Then $C$ has a stable model over $R$.

Proof. By Proposition 2.6 we know that the special fiber is tree-like. Now the claim is an immediate consequence of Liu2 chap.10, Prop.3.44.

As stated before, we shall always assume that the branch locus of $C \longrightarrow \mathbb{P}_{K}^{1}$ has equidistant geometry and consists of rational points. This has consequences for the image of the injection (2). Namely, any element of its image will have trivial action on the original component (cf. Definition 2.5) of the stably marked model. The group of such automorphisms we denote by $\operatorname{Aut}_{k}\left(C_{R^{\prime}} \otimes_{R^{\prime}} k\right)^{\#}$. Hence we have

$$
\operatorname{Gal}\left(K^{\prime} / K\right) \hookrightarrow \operatorname{Aut}_{k}\left(C_{R^{\prime}} \otimes_{R^{\prime}} k\right)^{\#}
$$

Definition 2.8. If the injection (3) is surjective we say that $C$ has maximal monodromy. If $v_{p}\left(\left|\operatorname{Gal}\left(K^{\prime} / K\right)\right|\right)=v_{p}\left(\left|\operatorname{Aut}_{k}\left(C_{R^{\prime}} \otimes_{R^{\prime}} k\right)^{\#}\right|\right)$ we say that $C$ has maximal wild monodromy.

The following section is the technical heart of the paper and introduces the monodromy polynomial.

\section{The monodromy polynomial}

For this section let $R$ be a discrete valuation ring as defined in section 2 and $f\left(X_{0}\right) \in$ $R\left[X_{0}\right]$ a monic polynomial of degree $n$ prime to the residue characteristic $p$ of $R$. Let $m$ be the number of distinct zeros of $f\left(X_{0}\right)$ in an algebraic closure of $\operatorname{frac}(R)$ and $r$ the greatest integer such that $r p<n$. For $X_{0}=X+Y$ Taylor expansion yields

$$
f\left(X_{0}\right)=f(X+Y)=s_{0}(Y)+s_{1}(Y) X+s_{2}(Y) X^{2}+\cdots+s_{n}(Y) X^{n} \in R[X, Y]
$$

which we view as a polynomial in two variables.

Definition 3.1. If $f\left(X_{0}\right)$ as above can be written

$$
f(X+Y)=s_{0}(Y)\left(H(X, Y)^{p}-\sum_{i=r+1}^{n} A_{i}(Y) X^{i}\right)
$$

with $H(X, Y)=1+a_{1}(Y) X+a_{2}(Y) X^{2}+\cdots+a_{r}(Y) X^{r} \in K(Y)[X], A_{i}(Y) \in K(Y)$ and $r$ as introduced earlier, we call this a special decomposition of $f\left(X_{0}\right)$.

The existence and properties of such a decomposition are established in Lemma 3.3 We set $S_{1}(Y)=s_{1}(Y) / \operatorname{gcd}\left(s_{0}(Y), s_{1}(Y)\right)$ and $S_{0}=s_{0}(Y) / \operatorname{gcd}\left(s_{0}(Y), s_{1}(Y)\right)$. Then $S_{1}(Y), S_{0}(Y) \in R[Y], f^{\prime}(Y) / f(Y)=S_{1}(Y) / S_{0}(Y), \operatorname{deg}\left(S_{1}(Y)\right)=m-1$, $\operatorname{deg}\left(S_{0}(Y)\right)=m$ and $\left(S_{0}(Y), S_{1}(Y)\right)=1$. Also, for $t \in \mathbb{N}$, we will use the following notation

$$
\left(\frac{1}{p}\right)=\frac{\prod_{i=0}^{t-1}\left(\frac{1}{p}-i\right)}{}
$$


For later use we note that the $p$-adic value of these binomial coefficients is given by

$$
-v_{p}\left(\left(\begin{array}{c}
\frac{1}{p} \\
t
\end{array}\right)\right)=t+[t / p]+\left[t / p^{2}\right]+\ldots
$$

where $v_{p}(p)=1$.

Lemma 3.2. Let $n$ be a positive integer not divisible by $p$, and $r$ the greatest integer, such that $r p<n$. Consider the equivalence relation $\mathcal{R}$ on $M=\{1,2, \ldots, n\}$ that identifies $a$ with $b$ if there exists an integer $e \geq 0$ such that $a=p^{e} b$ or $b=p^{e} a$.

Then the set $M_{0}=\{r+1, r+2, \ldots, n\}$ is a minimal complete system of representatives for the quotient $M / \mathcal{R}$. In particular $M_{0}$ contains exactly one power $p^{\alpha}$ of $p$, characterized by $p^{\alpha}<n<p^{\alpha+1}$.

The proof is elementary and omitted.

Lemma 3.3. i) A special decomposition as given by equation (5) above exists and is unique.

ii) There exist $t_{i}(Y), T_{i}(Y) \in R[Y]$ such that

$$
a_{i}(Y)=\left(\begin{array}{c}
\frac{1}{p} \\
i
\end{array}\right) \frac{s_{1}(Y)^{i}+p t_{i}(Y)}{s_{0}(Y)^{i}}=\left(\begin{array}{c}
\frac{1}{p} \\
i
\end{array}\right) \frac{S_{1}(Y)^{i}+p T_{i}(Y)}{S_{0}(Y)^{i}} \quad \text { for } \quad 1 \leq i \leq r
$$

where $t_{1}(Y)=0$ and for $1<i \leq r, \operatorname{deg}\left(t_{i}(Y)\right)=i(n-1)$.

iii) There exist $c_{i} \in K, T(Y) \in R[Y]$ and $N_{i}(Y) \in R[Y]$ monic such that

$$
\begin{gathered}
A_{i}(Y)=c_{i} \frac{N_{i}(Y)}{s_{0}(Y)^{i}} \quad \text { for } r+1 \leq i \leq n \quad \text { and } \\
A_{p^{\alpha}}(Y)=-\left(\begin{array}{c}
\frac{1}{p} \\
p^{\alpha-1}
\end{array}\right)^{p} \frac{S_{1}(Y)^{p^{\alpha}}+p T(Y)}{S_{0}(Y)^{p^{\alpha}}} \quad \text { with } \quad v_{p}\left(c_{p^{\alpha}}\right)=v_{p}\left(\left(\begin{array}{c}
\frac{1}{p} \\
p^{\alpha-1}
\end{array}\right)^{p}\right) \leq 0
\end{gathered}
$$

where $p^{\alpha}$ is the power determined by Lemma 3.2 and for $\alpha=0$ we $\operatorname{set}\left(\begin{array}{l}\frac{1}{p} \\ \frac{1}{p}\end{array}\right)=1$.

Proof. i) First observe that for $r=0$ we can take $H(X, Y)=1$ and then the statement is true. The case of $r>0$ remains to be considered. One computes the functions $a_{i}(Y)$ recursively from equation (5) starting with $a_{1}(Y)=s_{1}(Y) /\left(p s_{0}(Y)\right)$. This requires only solving linear equations and therefore the existence and uniqueness are obvious.

For later use we shall show the following property: $S_{0}(Y)^{i} a_{i}(Y) \in K[Y]$ for $1 \leq$ $i \leq r$. We proceed by induction on $i$. For $i=1$ this is immediate from the expression for $a_{1}(Y)$ given above. We therefore assume that the property holds for all $i$ with $1 \leq i \leq l<r$. From (15) we get

$$
f(X+Y)=s_{0}(Y)\left(1+a_{1}(Y) X+\cdots+a_{l}(Y) X^{l}\right)^{p}+p s_{0}(Y) a_{l+1} X^{l+1} \quad\left(X^{l+2}\right) .
$$

Comparing the coefficients of $X^{l+1}$ on each side we get an expression for $a_{l+1}(Y)$. Now taking into account that $S_{0}(Y)^{l+1} s_{l+1}(Y) / s_{0}(Y) \in K[Y]$ the induction hypothesis yields $S_{0}(Y)^{l+1} a_{l+1}(Y) \in K[Y]$.

ii) To derive these properties, an alternate approach, such as that utilized under i) is more advantageous. From (5) we get the following identity in $K(Y)[[X]]$ :

$$
H(X, Y)=\left(\frac{f(X+Y)}{c(V)}-\sum^{n} A_{i}(Y) X^{i}\right)^{1 / p}
$$


$=\left(1+\frac{s_{1}(Y)}{s_{0}(Y)} X+\frac{s_{2}(Y)}{s_{0}(Y)} X^{2}+\cdots+\frac{s_{n}(Y)}{s_{0}(Y)} X^{n}-\sum_{i=r+1}^{n} A_{i}(Y) X^{i}\right)^{1 / p}=(1+F(X))^{1 / p}$

where

$$
F(X)=\sum_{i=1}^{n} \frac{s_{i}(Y)}{s_{0}(Y)} X^{i}-\sum_{i=r+1}^{n} A_{i}(Y) X^{i} \in X K(Y)[[X]]
$$

We can develop this in $K(Y)[[X]]$ to get $(1+F(X))^{1 / p}$ with value 1 at $X=0$ :

$$
1+\left(\begin{array}{c}
\frac{1}{p} \\
1
\end{array}\right) F(X)+\left(\begin{array}{c}
\frac{1}{p} \\
2
\end{array}\right) F(X)^{2}+\left(\begin{array}{c}
\frac{1}{p} \\
3
\end{array}\right) F(X)^{3}+\ldots
$$

Because $\operatorname{deg}_{X}(H(X, Y)) \leq r$, we can compute modulo $X^{r+1}$ and therefore only need to evaluate the following expansion

$$
1+\left(\begin{array}{c}
\frac{1}{p} \\
1
\end{array}\right) F_{0}(X)+\left(\begin{array}{c}
\frac{1}{p} \\
2
\end{array}\right) F_{0}(X)^{2}+\left(\begin{array}{c}
\frac{1}{p} \\
3
\end{array}\right) F_{0}(X)^{3}+\ldots \quad \text { where } F_{0}(X)=\sum_{i=1}^{n} \frac{s_{i}(Y)}{s_{0}(Y)} X^{i}
$$

From this one reads the coefficients of $H(X, Y)$. Notice that by (6)

$$
\left(\begin{array}{c}
\frac{1}{p} \\
t
\end{array}\right)^{-1}\left(\begin{array}{c}
\frac{1}{p} \\
s
\end{array}\right) \in p R \quad \text { for any two integers } t>s .
$$

This justifies the binomial coefficient in the first expression for $a_{i}(Y)$. Under i) we have seen that $S_{0}(Y)^{i} a_{i}(Y) \in K[Y]$. Further, $s_{1}(Y) / s_{0}(Y)=S_{1}(Y) / S_{0}(Y)$ which allows us to write the second expression given for $a_{i}(Y)$ with $T_{i}(Y)$ a polynomial.

iii) Now that an expression for $H(X, Y)$ is determined we can use (15) to compute the $A_{i}(Y)$. The expression $A_{i}(Y)=c_{i} \frac{N_{i}(Y)}{s_{0}(Y)^{i}}$ is then immediate. To obtain $A_{p^{\alpha}}(Y)$ observe that $p^{\alpha-1}<r+1<p^{\alpha}$. Therefore

$$
A_{p^{\alpha}}(Y)=\frac{s_{p^{\alpha}}(Y)}{s_{0}(Y)}-a_{p^{\alpha-1}}(Y)^{p}+\text { other terms }
$$

Using (66) it is straightforward to verify that the other terms and $s_{p^{\alpha}}(Y)$ all have higher divisibility by $p$ than $a_{p^{\alpha-1}}(Y)^{p}$. Like for the $a_{i}(Y)$ this allows us to write

$$
A_{p^{\alpha}}(Y)=-\left(\begin{array}{c}
\frac{1}{p} \\
p^{\alpha-1}
\end{array}\right) \frac{s_{1}(Y)^{p^{\alpha}}+p t_{p^{\alpha}}(Y)}{s_{0}(Y)^{p^{\alpha}}} \quad \text { with } t_{p^{\alpha}}(Y) \in R[Y]
$$

We proceed to get the expression for $A_{p^{\alpha}}(Y)$ given in the lemma. One can rewrite (17) as

$$
s_{p^{\alpha}}(Y)=s_{0}(Y)\left[H_{p^{\alpha}}(Y)+A_{p^{\alpha}}(Y)\right]
$$

where $H_{p^{\alpha}}(Y)$ is the coefficient of $X^{p^{\alpha}}$ in $H(X, Y)^{p}$. Notice that $S_{0}(Y)^{p^{\alpha}} H_{p^{\alpha}}(Y) \in$ $K[Y]$ by what was said under i). We hence conclude that $S_{0}(Y)^{p^{\alpha}} A_{p^{\alpha}}(Y) \in K[Y]$. This yields the term $T(Y)$ in the formula for $A_{p^{\alpha}}(Y)$.

Definition 3.4. In the situation of Lemma 3.3 part iii) we define $\mathcal{L}(Y)=S_{1}(Y)^{p^{\alpha}}+$ $p T(Y)$. It is a polynomial of degree $p^{\alpha}(m-1)$ and we call it the monodromy polynomial of $f(Y)$.

From the definition one immediately obtains $\mathcal{L}(Y) \equiv S_{1}(Y)^{p^{\alpha}} \bmod p$. Notice that 


\section{Degeneration of $\mu_{p}$-torsors}

In this section we have collected various results on the degeneration of $\mu_{p}$-torsors. These are not new and similar results have appeared already in various places. For this reason we do not include proofs here but refer to the existing literature.

\subsection{The general case}

Let $R$ be a discrete valuation ring as in section 2 and recall the definition of $\lambda$ given there. Let $C \longrightarrow \mathbb{P}_{K}^{1}$ be a $p$-cyclic cover of smooth projective geometrically irreducible $K$-curves. Unlike in the rest of the paper, in section 4.1 we make no assumptions about the branch locus of the cover $C \longrightarrow \mathbb{P}_{K}^{1}$.

Definition 4.1. The valuation on $K\left(X_{0}\right)$ corresponding to the DVR $R\left[X_{0}\right]_{(\pi)}$ is called the Gauss valuation $v_{X_{0}}$ with respect to $X_{0}$.

$$
\text { We then have } v_{X_{0}}\left(\sum_{i=0}^{m} a_{i} X_{0}^{i}\right)=\min \left\{v\left(a_{i}\right) \mid 0 \leq i \leq m\right\} .
$$

Notice that a change of coordinates $X=\left(X_{0}-y\right) / \rho$ for $\rho, y \in R$ gives rise to a Gauss valuation $v_{X}$. It is equivalent to $v_{X_{0}}$ iff $\rho$ is a unit. Further, these valuations are exactly those that come from the local rings at generic points of components in the semi-stable models for $\mathbb{P}_{K}^{1}$.

Lemma 4.2. Let $C \longrightarrow \mathbb{P}_{K}^{1}$ be a p-cyclic cover of curves and $\operatorname{Proj}(R[u, w])$, with $X=u / w$, a smooth $R$-model for $\mathbb{P}_{K}^{1}$. Denote by $\mathcal{C}_{R}$ its normalization in $K(C)$. Suppose the cover is given birationally by an equation $Z^{p}=f(X)$ with $f(X) \in R[X]$ and such that $\pi$ does not divide $f(X)$. Then the cover of special fibers $\mathcal{C}_{R} \otimes_{R} k \longrightarrow$ $\operatorname{Proj}(k[u, w])$ is separable and irreducible iff

$$
\max \left\{v_{X}\left(h(X)^{p}-f(X)\right) \text { for } h(X) \in R[X]\right\}=v\left(\lambda^{p}\right) .
$$

The proof is elementary and essentially that of [Le] Prop.1 or Gr-Ma] III.Prop.1.1.

\subsection{The equidistant case}

In this section we work under the hypotheses of section 2 , i.e. let $C \longrightarrow \mathbb{P}_{K}^{1}$ be a $p$-cyclic cover and assume that the branch locus $B$ consists of $K$-points having equidistant geometry with respect to the coordinate $X_{0}$. We suppose that the cover is given by $Z_{0}^{p}=f\left(X_{0}\right)$ where $X_{0}=u / w$ and with $f\left(X_{0}\right) \in R\left[X_{0}\right]$ monic of degree $n$ prime to $p$. We will also use the notation introduced in Definition 3.1.

Remark 4.3. a) In the above situation, consider the flat projective $R$-model $\mathcal{C}_{R}$ for $C$ obtained by normalizing $\operatorname{Proj}(R[u, w])$ in the function field $K(C)$. From $\mathcal{C}_{R}$ we obtain the stably marked model $C_{R^{\prime}}$ for $C$ by a series of blowups, after passing to a finite extension $R^{\prime}$. The uniqueness of $C_{R^{\prime}}$ implies that the $p$-cyclic group action on $C$ extends to $C_{R^{\prime}}$ and the quotient by this action is a semi-stable model for $\mathbb{P}_{K^{\prime}}^{1}$, where $K^{\prime}=\operatorname{frac}\left(R^{\prime}\right)$ (cf. Ra1] appendice). This semi-stable model is obtained from $\operatorname{Proj}\left(R^{\prime}[u, w]\right)$ by a series of blowups. By construction, these are all centered on the affine patch $\operatorname{Spec}\left(R^{\prime}\left[X_{0}\right]\right)$ and hence correspond to ideals of the form $\left(X_{0}-y, \rho\right) \subset$ $R^{\prime}\left[X_{0}\right]$ We call $u$ the center and $o$ the radius and often sneak of the Gauss valuation 
$v_{X}$ with $X=\left(X_{0}-y\right) / \rho$ instead. All blowups we will consider are of this type and on $\operatorname{Proj}\left(R^{\text {alg }}[u, w]\right)$. Blowing up $\left(X_{0}-y, \rho\right) \subset R^{\prime}\left[X_{0}\right]$ gives an exceptional divisor which in turn, by normalization in $K^{\prime}(C)$, yields irreducible components of the stably marked model. The DVRs at their generic points are the ones that dominate the DVR of $v_{X}$. An interesting case, when there is only one, is treated in Proposition 4.4.

A local computation shows that the centers specialize to points below the singularities (cusps) of $\mathcal{C}_{R} \otimes_{R} k$ and are outside the specialization of the branch locus. Their $X_{0^{-}}$ coordinates are zeros of $\bar{f}^{\prime}\left(X_{0}\right)$, hence also zeros of $\bar{S}_{1}\left(X_{0}\right)=\bar{f}^{\prime}\left(X_{0}\right) / \operatorname{gcd}\left(\bar{f}^{\prime}\left(X_{0}\right), \bar{f}\left(X_{0}\right)\right)$. In particular, if $X_{0}=y$ is such a center, then $y \in R^{a l g}$ and $\bar{f}(\bar{y}) \in k-\{0\}$. A schematic picture of this situation is given in figure 1.

b) It is immediately derived from the equation that the cover of special fibers $\mathcal{C}_{R} \otimes_{R} k \longrightarrow \mathbb{P}_{k}^{1}$ is purely inseparable. Therefore, to yield a component of genus $>0$ in the stable reduction of $C$, a blowup (on $\operatorname{Proj}(R[u, w]))$ must have a radius $\rho$ with $v(\rho)>0$.
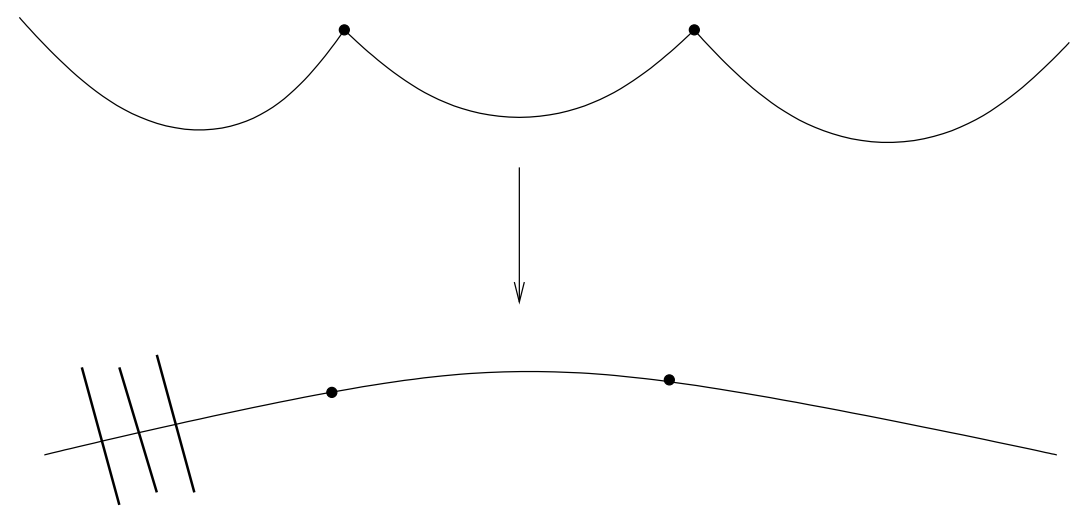

Figure 1: $\mathcal{C}_{R^{\prime}} \otimes_{R^{\prime}} k \longrightarrow \mathbb{P}_{k}^{1}$ with singularities and branch locus

With the above notation we obtain the following.

Proposition 4.4. The Gauss valuation corresponding to $X=\left(X_{0}-y\right) / \rho$ for $\rho, y \in$ $R^{\text {alg }}, \bar{f}(\bar{y}) \neq 0$ induces a separable and irreducible component in a semi-stable model of $C$ iff the following two conditions hold:

i)

$$
H(\rho X, y) \in R^{a l g}[X]
$$

ii)

$$
v_{X}\left(\frac{f(\rho X+y)}{s_{0}(y)}-H(\rho X, y)^{p}\right)=v\left(\lambda^{p}\right) .
$$

In this case, and with the change of coordinates $Z_{0} /\left(s_{0}(y)^{1 / p}\right)=\lambda Z_{1}+H(\rho X, y)$, the equation $Z_{0}^{p}=f\left(X_{0}\right)$ yields

$$
\begin{aligned}
& Z_{1}^{p}+\frac{p}{\lambda} Z_{1}^{p-1} H(\rho X, y)+\frac{\left(\begin{array}{c}
p \\
2
\end{array}\right)}{\lambda^{2}} Z_{1}^{p-2} H(\rho X, y)^{2}+\ldots \\
& +\frac{p}{\lambda^{p-1}} Z_{1} H(\rho X, y)^{p-1}-\frac{1}{\lambda^{p}} \sum_{i=r+1}^{n} A_{i}(y) \rho^{i} X^{i}=0
\end{aligned}
$$


which, for $v(\rho)>0$, in reduction gives the irreducible Artin-Schreier equation

$$
Z_{1}^{p}-Z_{1}-\left(\frac{1}{\lambda^{p}} \sum_{i=r+1}^{n} A_{i}(y) \rho^{i} X^{i}\right)^{-}=0
$$

defining an étale cover of $\mathbb{A}_{k}^{1}$.

Proof. This is a straightforward translation of Lemma 4.2 into the terminology of Definition 3.1. We therefore omit the details of the proof.

\section{Construction of a stably marked model}

Now we can state the theorem describing a stably marked model. We work under the hypotheses of section 2. Suppose that the cover is given birationally by $Z_{0}^{p}=f\left(X_{0}\right)$ with $f\left(X_{0}\right)$ monic of degree $n$ prime to $p$ and such that, with respect to $X_{0}$, the branch points have distinct specializations. Let $r$ be the greatest integer such that $r p<n$ and let $\alpha$ be determined by $p^{\alpha}<n<p^{\alpha+1}$. We write $|B|-1=m$ and can assume that $f\left(X_{0}\right)$ has been chosen such that $m(p-1) \geq n$. Choosing $f\left(X_{0}\right)$ this way limits the size of $\mathcal{L}(Y)$. We point out that, due to the freedom in choosing $f\left(X_{0}\right)$, the polynomial $\mathcal{L}(Y)$ is not uniquely determined by the cover $C \longrightarrow \mathbb{P}_{K}^{1}$.

Theorem 5.1. The components of genus $>0$ of the stably marked model of $C$ correspond bijectively to the Gauss valuations $v_{X_{j}}$ with $\rho_{j} X_{j}=X_{0}-y_{j}$ where $y_{j}$ is a zero of the monodromy polynomial $\mathcal{L}(Y)$ and $\rho_{j} \in R^{\text {alg }}$ is such that

$$
v\left(\rho_{j}\right)=\max \left\{\frac{1}{i} v\left(\frac{\lambda^{p}}{A_{i}\left(y_{j}\right)}\right) \text { for } r+1 \leq i \leq n\right\} .
$$

The dual graph of the special fiber of the stably marked model of $C$ is an oriented tree whose ends are in bijection with the components of genus $>0$.

Proof. The fact that the special fiber is tree-like is a consequence of Proposition 2.6. The statement about the ends follows from Ra2 2.4.8. Let $y_{j}$ be a zero of $\mathcal{L}(Y)$. Then $\overline{S_{1}\left(y_{j}\right)}=0$ and hence $y_{j} \in R^{\text {alg }}$. As $S_{1}(Y)$ and $s_{0}(Y)$ are relatively prime we conclude that $s_{0}\left(y_{j}\right)=f\left(y_{j}\right)$ is a unit (cf. Remark 4.3). We will show that for $\rho_{j}$ as defined above, one obtains a component of genus $>0$. As a first step, we need to show that $v\left(\rho_{j}\right)>0$. Therefore assume $v\left(\rho_{j}\right) \leq 0$. Then we get $v\left(A_{i}\left(y_{j}\right)\right) \geq v\left(\lambda^{p}\right)$ for all $r+1 \leq i \leq n$. Using equation (15) of Definition [3.1, we conclude that $H\left(X, y_{j}\right) \in R\left[y_{j}\right][X]$ and therefore equation (9) in Proposition 4.4 applies to show that the Gauss valuation corresponding to $\rho=1$ and $y=y_{j}$ induces a separable (not necessarily irreducible) component in a semi-stable reduction of $C$. Now this Gauss valuation is $\mathrm{PGL}_{2}\left(R\left[y_{j}\right]\right)$-equivalent to the one given by $X_{0}$, so both induce the same component in the semi-stable reduction. This is a contradiction, as we know that the latter induces a $\mu_{p}$-torsor. We conclude $v\left(\rho_{j}\right)>0$. Next consider the equation (8) in Proposition 4.4 for $y=y_{j}$ and $\rho=\rho_{j}$ :

$$
\begin{gathered}
Z_{1}^{p}+\frac{p}{\lambda} Z_{1}^{p-1} H\left(\rho_{j} X, y_{j}\right)+\frac{p}{\lambda^{2}} Z_{1}^{p-2} H\left(\rho_{j} X, y_{j}\right)^{2}+\ldots \\
-Z_{1} H\left(\rho_{j} X, y_{j}\right)^{p-1}-\frac{1}{\lambda p} \sum^{n} A_{i}\left(y_{j}\right) \rho_{j}^{i} X^{i}=0 .
\end{gathered}
$$


Then, by definition of $\rho_{j}$, the polynomial $\frac{1}{\lambda^{p}} \sum_{i=r+1}^{n} A_{i}\left(y_{j}\right) \rho_{j}^{i} X^{i}$ is in $R\left[y_{j}, \rho_{j}\right][X]$ and $s_{0}\left(y_{j}\right)$ is a unit as the branch locus has equidistant geometry. Now the relation (51) implies the same for $H\left(\rho_{j} X, y_{j}\right)$. Altogether we see that the above equation is in $R\left[y_{j}, \rho_{j}\right]\left[X, Z_{1}\right]$. Now, taking into account Proposition 4.4, we conclude that in reduction we get an irreducible Artin-Schreier equation. Further, using Artin-Schreier theory, Lemma 3.2 implies that the equation cannot have genus zero, because by definition of $\mathcal{L}(Y)$ we have $A_{p^{\alpha}}\left(y_{j}\right)=0$. This fact is the motivation for the definition of $\mathcal{L}(Y)$.

Conversely, suppose the Gauss valuation corresponding to $X=\left(X_{0}-y\right) / \rho$ induces a component of genus $>0$ in the stable reduction of $C$. Then, by Remark 4.3. $y \in R^{\text {alg, }}$, $v(\rho)>0$ and $s_{0}(y) \in R^{\text {alg }}$ is a unit. By Proposition 4.4 an Artin-Schreier equation for the component will be given by reducing equation (8) to $k$. By assumption the conductor of this equation is strictly bigger than 1 and in turn corresponds by Lemma 3.2 to a unique integer $t$ with $r+1 \leq t \leq n$ and $t \neq p^{\alpha}$. Now for any $y_{1}$ with $v\left(y_{1}-y\right) \geq v(\rho)$, the Gauss valuation corresponding to $X_{1}=\left(X_{0}-y_{1}\right) / \rho$ yields the same component in the stable reduction. In particular the associated Artin-Schreier equation has to have the same conductor, and hence the same value of $t$, as defined above. We conclude that $v\left(A_{t}(y)\right)=v\left(A_{t}\left(y_{1}\right)\right)$ and $v\left(A_{t}(y) \rho^{t}\right)=v\left(\lambda^{p}\right)$ for any $y_{1}$ with $v\left(y_{1}-y\right) \geq v(\rho)$. Using Lemma 3.3 iii) and observing that $s_{0}(y)$ and $s_{0}\left(y_{1}\right)$ are units we get $v\left(N_{t}(y)\right)=v\left(N_{t}\left(y_{1}\right)\right)$.

This implies that for all zeros $z$ of $N_{t}(Y)$ we have $v(z-y)<v(\rho)$. In particular there exist a $\rho^{\prime} \in R^{\text {alg }}$ such that $v(z-y)<v\left(\rho^{\prime}\right)<v(\rho)$ as $N_{t}(Y)$ has only finitely many zeros. Furthermore we can assume that $\rho^{\prime}$ has been chosen such that for all $\tilde{y}$ with $v(\rho)>v(y-\tilde{y})>v\left(\rho^{\prime}\right)$ the point $\tilde{y}$ is not a center for a component of genus $>0$ in the stable reduction of $C$. We fix $\tilde{y}$ such that $v(\rho)>v(y-\tilde{y})>v\left(\rho^{\prime}\right)$. Now pick $\tilde{\rho} \in R^{\text {alg }}$ such that

$$
v(\tilde{\rho})=\max \left\{\frac{1}{i} v\left(\frac{\lambda^{p}}{A_{i}(\tilde{y})}\right) \text { for } r+1 \leq i \leq n\right\} .
$$

By choice of $\rho^{\prime}$ we have $v(z-\tilde{y})<v\left(\rho^{\prime}\right)$ for all zeros $z$ of $N_{t}(Y)$. Therefore, as $v(y-\tilde{y})>v\left(\rho^{\prime}\right)$, we get $v\left(N_{t}(y)\right)=v\left(N_{t}(\tilde{y})\right)$. Now the definition of $\tilde{\rho}$ yields $v(\tilde{\rho}) \geq$ $v(\rho)$. Also by construction equation (8) of Proposition 4.4 for $y=\tilde{y}$ and $\rho=\tilde{\rho}$ will have integral coefficients and be irreducible in reduction. We already know that the corresponding component in a semi-stable reduction must have genus zero. Therefore $v\left(A_{t}(y)\right)=v\left(A_{t}(\tilde{y})\right)$ even implies $v(\tilde{\rho})>v(\rho)$. Further, using Lemma 3.3 iii), genus zero implies

$$
v\left(\left(\begin{array}{c}
\frac{1}{p} \\
p^{\alpha-1}
\end{array}\right)^{p} \mathcal{L}(\tilde{y}) \tilde{\rho}^{p^{\alpha}}\right)=v\left(\lambda^{p}\right) .
$$

By assumption the component corresponding to $X=\left(X_{0}-y\right) / \rho$ has genus $>0$ consequently

$$
v\left(\left(\begin{array}{c}
\frac{1}{p} \\
p^{\alpha-1}
\end{array}\right)^{p} \mathcal{L}(y) \rho^{p^{\alpha}}\right) \geq v\left(\lambda^{p}\right) .
$$

Altogether one concludes that

$$
v(\mathcal{L}(\tilde{y}))<v(\mathcal{L}(y)) \text { for all } \tilde{y} \text { with } \quad v\left(\rho^{\prime}\right)<v(y-\tilde{y})<v(\rho) .
$$

We claim this implies that there exists a zero $z$ of $\mathcal{L}(Y)$ with $v(z-y) \geq v(\rho)$. To see this assume the contrary, namely $v(z-y)<v(\rho)$ for all zeros $z$ of $\mathcal{L}(Y)$. Chose $\tilde{y}$ such 
possible because $\mathcal{L}(Y)$ has only finitely many zeros. Then $v(z-\tilde{y})=v(z-y+y-\tilde{y})=$ $v(z-y)$ for all zeros $z$ of $\mathcal{L}(Y)$ and hence $v(\mathcal{L}(y))=v(\mathcal{L}(\tilde{y}))$. This is a contradiction thus proving the claim.

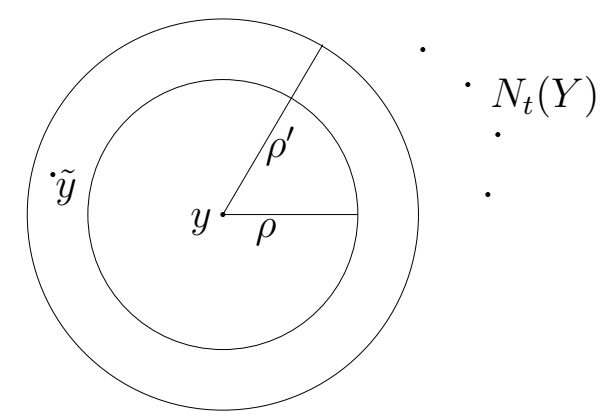

Figure 2: Illustrating the $p$-adic geometry in the second part of the proof of Theorem 5.1

Corollary 5.2. Let $K^{\prime} / K$ be the minimal extension such that $C_{K^{\prime}}$ has a stable model over the integral closure $R^{\prime}$ of $R$ in $K^{\prime}$ and denote by $F$ the splitting field of $\mathcal{L}(Y)$ over $K$. Let $E$ be the field obtained from $F$ by adjoining $\rho_{i}$ as well $\left(s_{0}\left(y_{i}\right)\right)^{1 / p}$ for each $i$. Then $K^{\prime} \subseteq E$. In particular $C$ has a stable model over the integral closure of $R$ in E.

Proof. Observe that all the blowups needed to obtain a stably marked model are defined over $E$, and the sum of the genera of the exceptional divisors is equal to the genus of the generic fiber (cf. Proposition 2.6). Now, applying Proposition 2.7 the result follows.

Remark 5.3. Assume that $C$ has potentially good reduction. Then the special fiber of the stably marked model $C_{R^{\prime}} \otimes_{R^{\prime}} k$ has 2 irreducible components: the original component and a component $E_{k}$ with the same geometric genus as $C$. We denote their unique point of intersection by $\infty$. By Proposition $4.4 E_{k}$ is a $p$-cyclic cover of $\mathbb{P}_{k}^{1}$ which is ramified only at $\infty$. Let $\operatorname{Aut}\left(E_{k}\right)_{\infty}$ be the group of automorphisms of $E_{k}$ that leave the point $\infty$ fixed. Then this group is isomorphic to the group $\operatorname{Aut}_{k}\left(C_{R^{\prime}} \otimes_{R^{\prime}} k\right)^{\#}$ which was defined above Definition 2.8, Let $q$ be the highest power of $p$ dividing $\left[K^{\prime}: K\right]$. From the injection (3) we conclude that $q$ also divides the order of the $p$-Sylow subgroup of $\operatorname{Aut}\left(E_{k}\right)_{\infty}$. Let $m=|B|-1$ and write $m=l p^{s}+d$ with $(l, p)=1$ and $1 \leq d \leq p-1$. Then using [Le-Ma1] Theorem 1.1 we get the following

1) If $d \neq 1$ then $\left|\operatorname{Aut}\left(E_{k}\right)_{\infty}\right|$ is at most divisible by $p$ hence $q \in\{1, p\}$ (cf. [Le-Ma1, Thm.1 II.b). This explains why in Le Theorem 1 we got a criterion for good reduction with $\left[K^{\prime}: K\right]$ at most divisible by $p$.

2) If $d=1$ and $l>1$ then $\left|\operatorname{Aut}\left(E_{k}\right)_{\infty}\right|$ is at most divisible by $p^{s+1}\left(p^{s}\right.$ if $\left.p=2\right)$. Further the $p$-Sylow subgroup of $\operatorname{Aut}\left(E_{k}\right)_{\infty}$ injects into an extraspecial $p$-group (cf. Le-Ma1], Thm.1 II.d and Su] chap. 4 for the notion of extraspecial group). In this case, by Definition [3.4 the polynomial $\mathcal{L}(Y)$ has degree $l p^{s+\alpha}$ (recall that $\alpha$ is defined by $\left.p^{\alpha}<n<p^{\alpha+1}\right)$. 
3) If $d=l=1$ then $\left|\operatorname{Aut}\left(E_{k}\right)_{\infty}\right|$ is at most divisible by $p^{2 s+1}$. Again the $p$-Sylow subgroup of $\operatorname{Aut}\left(E_{k}\right)_{\infty}$ injects into an extraspecial $p$-group (cf. [Le-Ma1, Thm.1 II.a). Recall that $n=\operatorname{deg}\left(f\left(X_{0}\right)\right)$ and $m \leq n \leq(p-1) m$. Using $m=1+p^{s}$ we get $p^{s}<n<p^{s+1}$ and Definition 3.4 shows that $\mathcal{L}(Y)$ has degree $p^{2 s}$.

Fix $n$ and $m=p^{s}+1$. Point 3), together with Example 5.5 below, show that $\mathcal{L}(Y)$ has the smallest degree one could expect for this $n$ and $m$, as it has been defined independently of the type of degeneration of the model.

Remark 5.4. As explained earlier, there are bounds for the finite monodromy group $\operatorname{Gal}\left(K^{\prime} / K\right)$ derived from the injection (3) and the bounds for automorphism groups in positive characteristic (cf. [Le-Ma1 Thm.1). Examples 5.5 and 5.6 give reason to believe that these bounds on $\operatorname{Gal}\left(K^{\prime} / K\right)$ are sharp, as there we have maximal wild monodromy. Many more examples of this type will be given in forthcoming work of the authors (cf. [Le-Ma2]). Due to the relationship between $\mathcal{L}(Y)$ and $K^{\prime} / K$ given by Thm 5.1 and Cor 5.2 , the degree of $\mathcal{L}(Y)$ should reflect these bounds, if the degree of $\mathcal{L}(Y)$ is optimal. We fix $n$ and $m$.

a) Remark 5.3 above shows that for $m=1+p^{s}$ this is the case.

b) For $m=l p^{s}+d,(l, p)=1,1 \leq d \leq p-1$ : Assume there exists a cover $C \longrightarrow \mathbb{P}_{K}^{1}$ whose stable reduction contains a component $E$ of genus $p^{a}(p-1) / 2$ with $a=\left[\log _{p}(m-1)\right]$ maximal. $E$ is a $p$-cyclic cover of $\mathbb{P}_{k}^{1}$ that is ramified in exactly one point that we denote $\infty$. The assumption on the genus of $E$ is hence equivalent to the conductor of this cover at $\infty$ being equal to $p^{a}+1$. Further suppose that $C$ has maximal wild monodromy (cf. Def 2.8). A small calculation shows that, with few exceptions that we will not look at here, $a=\alpha$, where $p^{\alpha}<n<p^{\alpha+1}$. [Le-Ma1] Thm.1 yields $v_{p}(|\operatorname{Aut}(E)|) \leq 2 \alpha+1$ where equality is possible. On the other hand $\operatorname{deg}(\mathcal{L}(Y))=(m-1) p^{\alpha}$ and $p^{\alpha} \leq m-1<p^{\alpha+1}$ imply $p^{2 \alpha} \leq \operatorname{deg}(\mathcal{L}(Y))<p^{2 \alpha+1}$. Comparing with the size of $v_{p}(|\operatorname{Aut}(E)|)$ we see that $v_{p}(\operatorname{deg}(\mathcal{L}(Y)))$ is optimal if one makes the above assumptions on the existence of certain covers with prescribed reduction.

Notice that the discrepancy of 1 between the right hand side of $v_{p}(|\operatorname{Aut}(E)|) \leq$ $2 \alpha+1$ and the left hand side of $2 \alpha<v_{p}(\operatorname{deg}(\mathcal{L}(Y)))$ is explained by the fact that in Cor 5.2 certain $p$-th roots have to be adjoined.

We finish by giving some indication on our examples of maximal finite monodromy over $\mathbb{Q}_{p}^{\text {tame }}$. A more extensive version of this will be published elsewhere (cf. [Le-Ma2]).

Example 5.5 (Potentially good reduction with $m=1+p^{s}$ ). Let $p>2, q=p^{n}$, $n \geq 1, K=\mathbb{Q}_{p}^{\text {ur }}\left(p^{p /(q+1)}\right)$ and $C \longrightarrow \mathbb{P}_{K}^{1}$ be given birationally by the equation

$$
Z_{0}^{p}=f\left(X_{0}\right)=1+p^{p /(q+1)} X_{0}^{q}+X_{0}^{q+1} .
$$

Then $C$ has potentially good reduction and $\mathcal{L}(Y)$ is irreducible over $K$. The finite monodromy extension $K^{\prime} / K$ is obtained from the splitting field of $\mathcal{L}(Y)$ by adjoining $f(y)^{1 / p}$ for $y$ any zero of $\mathcal{L}(Y)$. It is Galois with the extraspecial group of exponent $p$ and order $p q^{2}$ (see $[\mathrm{Su}]$ ch. 4 for these groups). Further the finite monodromy is maximal in the sense of Definition 2.8.

Example 5.6 (Genus 2-curves and wild monodromy). Now we consider the case of $p$-cyclic covers where $p=2$ and $m=5$, i.e. genus 2 curves over a 2 -adic field in $\mathbb{Q}_{2}^{\text {tame }}$. In this case, there are three possible types for the degeneration of the stably 
by $Z_{0}^{p}=f\left(X_{0}\right)$ and can choose $f\left(X_{0}\right)$ of the form $f\left(X_{0}\right)=1+b_{2} X_{0}^{2}+b_{3} X_{0}^{3}+b_{4} X_{0}^{4}+$ $X_{0}^{5} \in R\left[X_{0}\right]$. We write $Q_{8}$ for the quaternion group and $D_{8}$ for the dihedral group of order 8 .

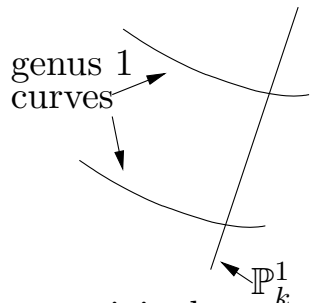

original component

Type 1

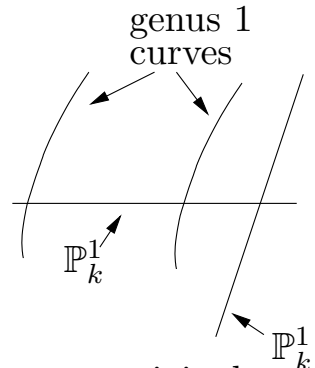

original component

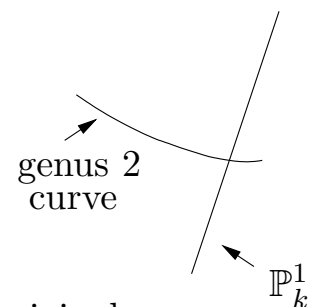

original component

Type 2

Type 3

$\operatorname{Gal}\left(K^{\prime} / K\right)_{w} \hookrightarrow Q_{8} * D_{8}$

Figure 3: The three types of degeneration

We will see that the wild monodromy can be maximal in any of the three types of degeneration.

a) Let $f\left(X_{0}\right)=1+2^{3 / 5} X_{0}^{2}+X_{0}^{3}+2^{2 / 5} X_{0}^{4}+X_{0}^{5}$ and $K=\mathbb{Q}_{2}^{\text {ur }}\left(2^{1 / 15}\right)$. Using our Theorem [5.1] and Magma calculations one shows that $C$ has stably marked reduction of type 1. Further the finite monodromy extension is maximal; its Galois group is $Q_{8} \times Q_{8}$.

b) Let $K=\mathbb{Q}_{2}^{\text {ur }}(a)$ with $a^{9}=2$. Consider the cover $C \longrightarrow \mathbb{P}_{K}^{1}$ given by

$$
Z_{0}^{2}=f\left(X_{0}\right)=1+a^{3} X_{0}^{2}+a^{6} X_{0}^{3}+X_{0}^{5}
$$

This cover has stably marked reduction of type 2 and maximal finite monodromy. Its Galois group is isomorphic to $\left(Q_{8} \times Q_{8}\right) \rtimes \mathbb{Z} / 2 \mathbb{Z}$, with $\mathbb{Z} / 2 \mathbb{Z}$ acting by exchanging the two factors.

c) Let $C \longrightarrow \mathbb{P}_{K}^{1}$ be given by the Kummer equation $Z_{0}^{2}=f\left(X_{0}\right)=1+X_{0}^{4}+X_{0}^{5}$ with $K=\mathbb{Q}_{2}^{\text {ur }}$. This cover has potentially good reduction (i.e. is of type 3 ) and maximal wild monodromy with group the central product $Q_{8} * D_{8}$.

Remark 5.7. In Si-Za1] and Si-Za2] Silverberg and Zarhin study the finite monodromy groups for abelian surfaces. In particular, they classify the finite groups which can occur as finite monodromy groups for abelian surfaces. Their examples are mostly in equal characteristic, and they ask at the end of loc. cit. section 1 for examples in mixed characteristic. Our Example [5.6 is such a case.

Thanks. We would like to thank Qing Liu for illuminating discussions. Part of this paper was written while the second author was a guest of the Max-Planck-Institut in Bonn. We would also like to thank the referees for their constructive remarks concerning the presentation of the paper. 


\section{References}

[Ab] A. Abbes, Réduction semi-stable des courbes, Courbes semi-stables et groupe fondamental en géometrie algébrique, Progress in Mathematics 187, Birkhäuser (2000).

[Co] R. Coleman, Computing stable reductions, Séminaire de Théorie des Nombres, Paris 1985-86. Basel-Boston-Berlin: Birkhäuser (1987).

[Co-Mc] R. Coleman, W. McCallum, Stable reduction of Fermat curves and Jacobi sum Hecke characters, J. Reine Angew. Math. 385, pp.41-101 (1988).

[De-Mu] P. Deligne, D. Mumford, The irreducibility of the space of curves of given genus, Inst. Hautes Études Sci. Publ. Math. 36, pp. 75-109 (1969).

[Des] M. Deschamps, Réduction semi-stable, Pinceaux de courbes de genre au moins deux (L. Szpiro, ed.), Astérisque, vol. 86, pp.1-34 (1981).

[Gr-Ma] B. Green, M. Matignon, Order $p$ automorphisms of the open disc of a p-adic field, J. Amer. Math. Soc. 12, no. 1, pp.269-303 (1999).

[Kr] M. Krir, Degré d'une extension de $\mathbb{Q}_{p}^{\mathrm{ur}}$ sur laquelle $J_{0}(N)$ est semi-stable, Ann. Inst. Fourier 46 (1996), no. 2, 279-291.

[Le] C. Lehr, Reduction of p-cyclic covers of the projective line, Manuscripta Math. 106, 2, pp.151-175 (2001).

[Le-Ma1] C. Lehr, M. Matignon, Automorphism groups for p-cyclic covers of the affine line, Compos. Math. 141, no.5, pp.1213-1237 (2005)

[Le-Ma2] C. Lehr, M. Matignon, Maximal wild monodromy in unequal characteristic, Preprint (2006)

[Liu1] Q. Liu, Stable reduction of finite covers of curves, Compos. Math. 142, no.1, pp.101-118 (2006)

[Liu2] Q. Liu, Algebraic Geometry and Arithmetic Curves, Oxford Graduate Texts in Mathematics, 6, Oxford University Press (2002).

[Li-Lo] Q. Liu, D. Lorenzini, Models of curves and finite covers, Compositio Math. 118, no. 1, pp.61-102. (1999).

[Ma] M. Matignon, Vers un algorithme pour la réduction stable des revêtements p-cycliques de la droite projective sur un corps p-adique, Mathematische Annalen 325, pp.323-354 (2003).

[Ra1] M. Raynaud, p-groupes et réduction semi-stable des courbes, The Grothendieck Festschrift, Vol.3, Basel-Boston-Berlin: Birkhäuser (1990).

[Ra2] M. Raynaud, Spécialisation des revêtements en caractéristique $p>0$, Ann. Scient. Ec. Norm. Sup. 32(4), pp.87-126 (1999).

[Si-Za1] A. Silverberg, Yu. G. Zarhin, Subgroups of inertia groups arising from abelian varieties, J. Algebra 209, no. 1, pp.94-107 (1998). 
[Si-Za2] A. Silverberg, Yu. G. Zarhin, Inertia groups and abelian surfaces, J. of Number Theory 110, pp.178-198 (2005)

[Su] M. Suzuki, Group theory II, Grundlehren der Mathematischen Wissenschaften 248. Springer-Verlag, New York, 1986.

\section{Claus LEHR}

Dipartimento di Matematica Pura ed Applicata

Università degli Studi di Padova, Via G.Belzoni 7, 35131 Padova, Italia

e-mail : lehr@math.unipd.it

Michel MATIGNON

Laboratoire de Théorie des Nombres et d'Algorithmique Arithmétique, UMR 5465 CNRS

Université de Bordeaux I, 351 cours de la Libération, 33405 Talence Cedex, France e-mail : matignon@math.u-bordeaux1.fr 